\title{
Resultados del anclaje foveal artroscópico sin nudo del complejo del fibrocartílago triangular en lesiones Atzei 2/3
}

\author{
Matías Sala," Sergio Daroda" \\ *División Cirugía de Mano y Miembro Superior, Hospital Regional de Comodoro Rivadavia, Comodoro Rivadavia, Chubut, Argentina \\ ** Clínica de la Mano Gamma, La Plata, Buenos Aires, Argentina
}

\begin{abstract}
RESUMEN
Objetivo: Evaluar, en forma retrospectiva, la función y los resultados objetivos de los pacientes con lesión foveal del complejo del fibrocartílago triangular mediante anclaje óseo sin nudo artroscópico. Materiales y Métodos: Se evaluó, en forma retrospectiva, a 12 pacientes con lesión foveal del complejo del fibrocartílago triangular Atzei 2/3 y un seguimiento promedio de 18 meses. Todas las lesiones se repararon con artroscopia mediante la guía de fibrocartílago y anclaje óseo sin nudo. Se evaluaron los rangos de movilidad y la fuerza de agarre con la escala de muñeca de la Clínica Mayo y el cuestionario DASH. Resultados: Se obtuvieron mejoras en la prueba del dolor y la fuerza; el rango de movilidad promedio fue de $70^{\circ}$ de extensión, $85^{\circ}$ de flexión, $20^{\circ}$ de desviación radial y $30^{\circ}$ de desviación cubital. Los resultados según la escala de muñeca de la Clínica Mayo fueron excelentes en el $83 \%$ y buenos en el $17 \%$, no hubo resultados malos. El puntaje promedio del cuestionario DASH fue 56 en el preoperatorio y 8 en el posoperatorio. El promedio de la fuerza comparativa contralateral fue del $70 \%$. Conclusiones: La reparación artroscópica de las lesiones Atzei 2/3 mediante anclaje óseo sin nudo logra buenos resultados, mejora los rangos de movilidad, recupera la estabilidad en todos los casos y la fuerza total o parcial.
\end{abstract}

Palabras clave: Artroscopia de muñeca; Atzei 2; Atzei 3; complejo del fibrocartílago triangular; anclaje óseo sin nudo.

Nivel de Evidencia: IV

Results of the Knotless Arthroscopic Foveal Anchorage of the Triangular Fibrocartilage Complex in Atzei 2/3 Lesions

\begin{abstract}
Objective: To retrospectively evaluate the functional and objective outcomes in patients with foveal lesions of the triangular fibrocartilage complex treated with arthroscopic knotless bone anchors. Materials and Methods: We retrospectively evaluated 12 patients with Atzei $2 / 3$ foveal lesions of the triangular fibrocartilage complex with an average follow-up of 18 months. All the lesions were repaired arthroscopically using a fibrocartilage guide and knotless bone anchors. The ranges of motion and grip strength were assessed with the Mayo Clinic wrist scale and the DASH questionnaire. Results: Improvements were obtained in the pain and strength test; the average range of motion was $70^{\circ}$ in extension, $85^{\circ}$ in flexion, $20^{\circ}$ in radial deviation, and $30^{\circ}$ in ulnar deviation. The outcomes according to the Mayo Clinic wrist scale were excellent in $83 \%$ of the cases and good in $17 \%$; there were no poor outcomes. The average DASH score was 56 preoperatively and 8 postoperatively. The average contralateral comparative strength was $70 \%$. Conclusion: The arthroscopic repair of Atzei $2 / 3$ lesions with knotless bone anchors achieves good outcomes, improves ranges of motion, and restores stability and total or partial strength in all cases.
\end{abstract}

Keywords: Wrist arthroscopy; Atzei 2; Atzei 3; triangular fibrocartilage complex; knotless bone anchorage.

Level of Evidence: IV

Recibido el 13-5-2021. Aceptado luego de la evaluación el 11-7-2021 • Dr. MATíAS SALA • salajuanmatias@icloud.com (ID) https://orcid.org/0000-0001-5542-5004

Cómo citar este artículo: Sala M, Daroda S. Resultados del anclaje foveal artroscópico sin nudo del complejo del fibrocartilago triangular en lesiones Atzei 2/3. Rev Asoc Argent Ortop Traumatol 2021;86(5):581-594. https://doi.org/10.15417/issn. 1852-7434.2021.86.5.1374 


\section{INTRODUCCIÓN}

El complejo del fibrocartílago triangular (CFCT) es el principal estabilizador de la articulación radiocubital distal (ARCD) y está formado por el disco articular, los ligamentos dorsales y palmares proximales y distales, los ligamentos cubitocarpianos y el subcreatum ligamentus. La estabilidad de la ARCD depende del CFCT, la cápsula articular, el cubital posterior, el pronador cuadrado y la banda oblicua distal. El CFCT es un disco fibrocartilaginoso con una rica vascularización en su zona cubital, precaria en el lado radial y nula en el centro del disco. ${ }^{1,2}$ Este complejo está rodeado de estructuras fibrosas que componen una llave de rotación, carga y traslación de fuerzas de la muñeca hacia el antebrazo. ${ }^{1}$

La lesión del CFCT es una de las causas más comunes de dolor cubital de la muñeca, que limita las actividades de la vida diaria. Es frecuente en atletas con alto nivel de competencia, por trauma o uso repetitivo de la articulación. ${ }^{1,3}$ Es por eso que, en los últimos años, hay un gran interés en su reparación con técnicas de fijación resistente mediante túneles óseos y anclajes capsulares con técnicas de arpones y sin nudo. ${ }^{3}$

Los anclajes sin nudo son una opción válida para la fijación de estructuras ligamentarias en los huesos, con similares resultados a los de los anclajes con nudo. Una ventaja de los anclajes sin nudo es la técnica más rápida de fijación e igual de resistente que los anclajes con nudos. ${ }^{4,5}$

Palmer distinguió dos tipos de lesiones del CFCT, las traumáticas (agudas) y las degenerativas (crónicas) (Tabla 1). ${ }^{6}$ Las lesiones agudas ocurren por traumatismos de la muñeca con hiperextensión y desviación cubital, traumatismo directo o actividades que requieran cargar peso. ${ }^{1,3}$ Las lesiones degenerativas se producen por actividades repetitivas con carga axial y desviación cubital de la muñeca, así como por la mala consolidación de fracturas de radio distal..$^{-9}$

Tabla 1. Clasificación de Palmer para lesiones del complejo del fibrocartílago triangular
Tipo 1
A. Perforación central
B. Lesión del borde cubital
C. Lesión de ligamentos cubitocarpianos
D. Lesión del borde radial
Tipo 2
A. Desgaste central del CFCT
B. Desgaste central del CFCT más condromalacia del SL o la cabeza del cúbito, o de ambos
C. Perforación del CFCT y condromalacia del SL o la cabeza del cúbito, o de ambos
D. Igual que C con lesión ligamentaria lunopiramidal
E. Igual que D con artrosis cubitocarpiana

$\mathrm{CFCT}=$ complejo del fibrocartílago triangular, $\mathrm{SL}=$ semilunar.

Aztei y cols., por su parte, modificaron mediante su clasificación artroscópica el concepto de "hamaca" del CFCT por el de "iceberg", donde la parte visible son las fibras superficiales que toleran y absorben el impacto, y la parte no visible son las fibras profundas, responsables de la estabilidad de este complejo (Tabla 2). ${ }^{1,3}$

La artroscopia de muñeca es el método de elección para el diagnóstico y el tratamiento de las lesiones del CFCT. Las dos pruebas artroscópicas más utilizadas son la prueba del gancho (hook test) para las lesiones foveales y la prueba del trampolín (trampoline test) para las lesiones superficiales. La prueba del fantasma es otra herramienta útil que se realiza desde el portal radiocubital distal dorsal., ${ }^{2,8}$

La técnica de elección para tratar las lesiones con inestabilidad del CFCT consiste en la reinserción de las fibras foveales de este complejo. ${ }^{1,2,7,8}$

A pesar de que la cirugía abierta logra buenos resultados a largo plazo, las técnicas artroscópicas tienen las siguientes ventajas: se puede evaluar la lesión por completo y tratar las lesiones asociadas, y la rigidez posoperatoria y el daño capsular son menores. ${ }^{1,3}$

El objetivo de este estudio es comunicar los resultados funcionales de pacientes operados con lesiones del CFCT Atzei 2/3 mediante el anclaje artroscópico sin nudo, considerando que es una técnica fiable, resistente, rápida y de simples pasos. 
Tabla 2. Clasificación de Atzei para lesiones cubitales del complejo del fibrocartílago triangular sobre la base de su estabilidad, las estructuras ligamentarias, el potencial de reparación y el tratamiento sugerido

\begin{tabular}{|r|c|c|c|c|c|c|}
\hline Tipo & $\begin{array}{c}\text { Inestabilidad } \\
\text { RCD }\end{array}$ & $\begin{array}{c}\text { Aspecto } \\
\text { del CFCT } \\
\text { distal }\end{array}$ & $\begin{array}{r}\text { Aspecto } \\
\text { del CFCT } \\
\text { proximal }\end{array}$ & $\begin{array}{r}\text { Capacidad } \\
\text { de reparación } \\
\text { del CFCT }\end{array}$ & $\begin{array}{c}\text { Aspecto } \\
\text { del cartí- } \\
\text { lago RCD }\end{array}$ \\
\hline $\mathbf{1}$ & Leve /No & Roto & Intacto & Buena & Bueno & Reparación capsular \\
\hline $\mathbf{2}$ & Moderada /Severa & Roto & Roto & Buena & Bueno & Reparación foveal \\
\hline $\mathbf{3}$ & Moderada/Severa & Intacto & Roto & Buena & Bueno & Reparación foveal \\
\hline $\mathbf{4}$ & Severa & Roto & Roto & Pobre & Bueno & Reconstrucción con injerto \\
\hline $\mathbf{5}$ & Moderada/Severa & Variable & Variable & Variable & Pobre & Artroplastia o salvataje \\
\hline
\end{tabular}

$\mathrm{RCD}=$ radiocubital distal, $\mathrm{CFCT}=$ complejo del fibrocartílago triangular.

\section{MATERIALES Y MÉTODOS}

Se desarrolló un estudio retrospectivo para analizar los resultados quirúrgicos en 12 pacientes adultos consecutivos con lesiones profundas del CFCT Atzei 2/3, operados entre el 1 de enero de 2018 y el 31 de diciembre de 2019.

Los criterios de inclusión fueron: pacientes $>18$ años, deportistas, sin antecedentes de cirugía del CFCT ni artroscopia de muñeca, dolor en el borde cubital de la muñeca compatible con lesión del CFCT que limitaba el trabajo, el deporte o las actividades diarias, pruebas de Berger, Ruby y Nakamura positivas, lesiones Atzei 2/3 confirmadas por resonancia magnética y artroscopia, y un seguimiento posoperatorio mínimo de 12 meses. Los criterios de exclusión fueron: cirugía previa del CFCT, artroscopia previa de muñeca, lesiones degenerativas y artrosis de la ARCD. Se estudió a 12 pacientes (9 hombres y 3 mujeres, 10 diestros y 2 zurdos) con lesiones foveales inestables del CFCT.

Las lesiones ligamentarias del carpo asociadas se evaluaron con la clasificación de Geissler (Tabla 3). ${ }^{10}$

Tabla 3. Clasificación de Geissler

\begin{tabular}{|c|l}
\hline Tipos & \\
\hline $\mathbf{1}$ & $\begin{array}{l}\text { Atenuación o hemorragia vista por radiocarpiana sin incongruencia. Permite el paso del palpador de } 1 \mathrm{~mm} \text { por } \\
\text { mediocarpiana }\end{array}$ \\
\hline $\mathbf{2}$ & Rotura con leve incongruencia. Permite el paso del palpador por mediocarpiana sin rotación \\
\hline $\mathbf{3}$ & Rotura con incongruencia. Permite el paso del palpador por mediocarpiana con rotación \\
\hline $\mathbf{4}$ & Rotura con incongruencia. Permite el pasaje de la óptica desde mediocarpiano a radiocarpiano entre los huesos \\
\hline
\end{tabular}

En todos los pacientes, se evaluaron, en forma objetiva, el rango de movilidad de la muñeca, en flexión, extensión y en desviaciones cubital y radial mediante un goniómetro de mano PVS estándar y la fuerza de puño con un dinamómetro hidráulico estándar (Baseline-Orthowell®).

La evaluación subjetiva se realizó con la escala analógica visual para dolor, la escala de muñeca de la Clínica Mayo y el cuestionario DASH..$^{11,12}$

El protocolo de tratamiento fue el siguiente: ante la primera consulta por dolor cubital de la muñeca, se realiza la anamnesis sobre el mecanismo del trauma y luego se procede al examen clínico. Utilizamos las pruebas de compresión axial, de Waiter, de Ruby, de Berger y la de Nakamura en tres posiciones. ${ }^{13}$ Luego solicitamos radiografías anteroposteriores y con carga, una resonancia magnética sin contraste para confirmar el diagnóstico, e inmovilizamos al paciente con una férula tipo pinza de azúcar, por tres semanas. Al retirar la inmovilización, el paciente comienza con fisioterapia y repetimos las pruebas, si son positivas, indicamos la artroscopia diagnóstica y terapéutica de muñeca. Estos tiempos se basan en el estudio de Aztei quien indica la reparación antes de los tres meses posteriores a la lesión para obtener buenos resultados. 


\section{Técnica quirúrgica}

Se administra anestesia de bloqueo plexual y se coloca un manguito preventivo de isquemia a $250 \mathrm{mmHg}$. Se utilizan portales 3/4, 6R, 6U, MCU y MCR.

Se explora la articulación radiocarpiana y mediocarpiana en busca de lesiones asociadas. Luego se realizan las pruebas del gancho, del fantasma y del trampolín para el CFCT y se clasifica la lesión (Figura 1).

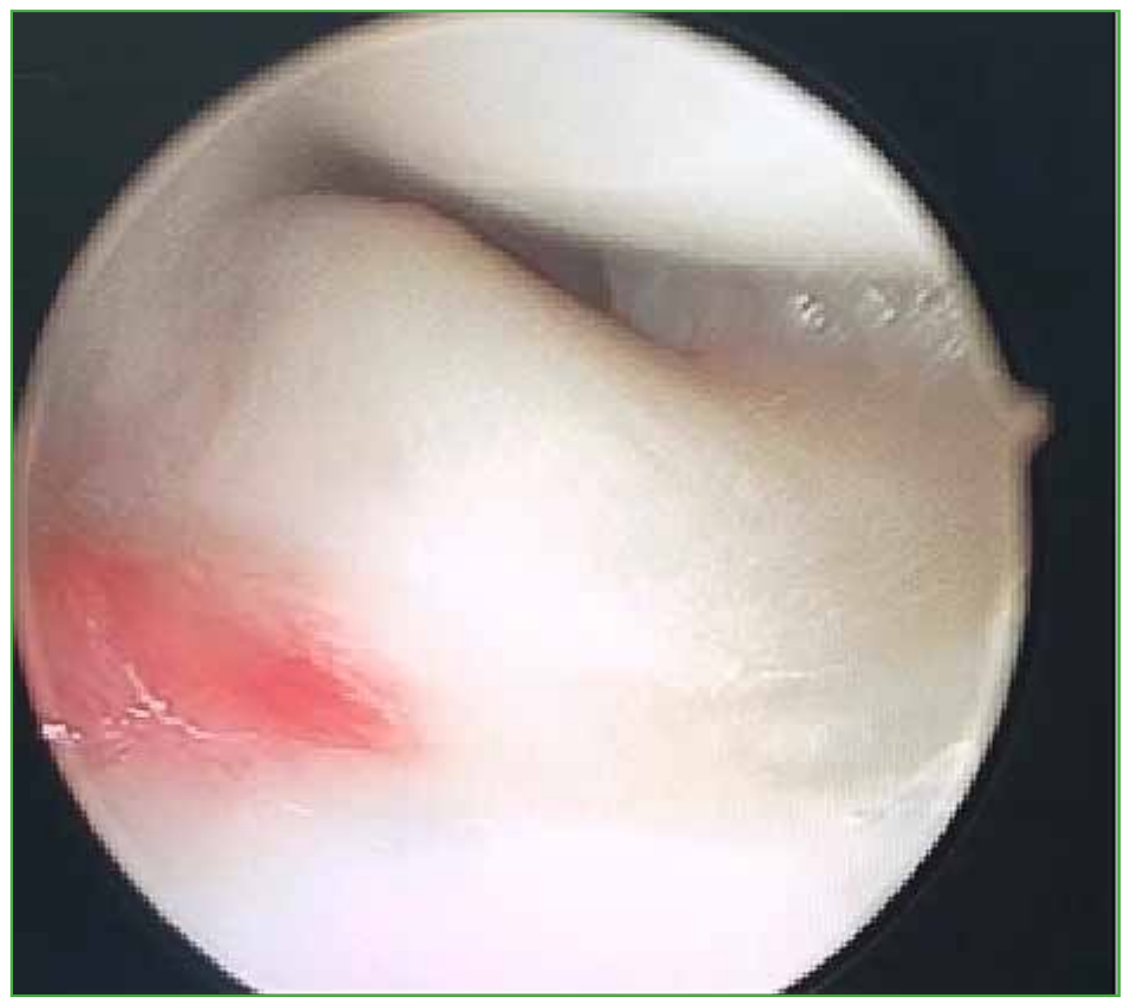

Figura 1. Prueba del fantasma positiva compatible con lesión foveal.

Se procede a la sinovectomía y el desbridamiento de la lesión (Figura 2). A continuación, se coloca la guía del CFCT por el portal 6R y bajo visión 3/4, se coloca la guía en la zona $2 / 2$ descrita por Matsumoto (Figura 3). Se efectúa un abordaje cubital de $2 \mathrm{~cm}$ para colocar la guía al mismo tiempo que se la posiciona por el portal $6 \mathrm{R}$ y se coloca una clavija por la guía hasta salir en la articulación y asegurarse de estar en la zona $2 / 2$. Se fresa con una guía de 3.0 desde el cúbito hasta la articulación. Al finalizar se retira la guía y se coloca por el orificio cubital un pasador de hilo 2.0 tipo fiberwire penetrando el disco; se retira ese hilo por el portal 6R sin perderlo por el túnel cubital (Figuras 4-6). A continuación, se coloca un pasador de hilo con un rescatador de sutura por el túnel cubital penetrando el disco $2 \mathrm{~mm}$ a volar o dorsal y se retira el rescatador de sutura por el portal 6R (Figuras 7-9). Se pasa el hilo por el rescatador de sutura y se tira desde el rescatador de sutura por el abordaje cubital siempre mirando desde portal 3/4. Se observa cómo la sutura pasa hacia el orificio cubital tensando el fibrocartílago (Figuras 10-12). Mediante un dispositivo de anclaje sin nudo de $2,8 \mathrm{~mm}$ se colocan las dos puntas de los hilos dentro del sistema de fijación y se lo impacta $5 \mathrm{~mm}$ distal al túnel realizado (Figuras 13 y 14). Se comprueba que las pruebas del trampolín y del gancho sean negativas, se retira la tracción y se prueba la estabilidad. Se cierran los portales. Se coloca una férula tipo Munster (pinza de azúcar) por cuatro semanas con movilidad de dedos y hombro, y luego, se inicia el programa de rehabilitación. Las primeras dos semanas se realiza con férula corta ballenada de muñeca de uso permanente, solo se retira la férula en rehabilitación y dentro de la casa para una movilidad controlada de la muñeca. Luego de las seis semanas, se usa la férula rígida de muñeca por la noche únicamente y se indica la movilidad enérgica de la muñeca libre sin carga. A las ocho semanas, se comienza con la fuerza progresiva y se retira, en forma definitiva, la muñequera. 


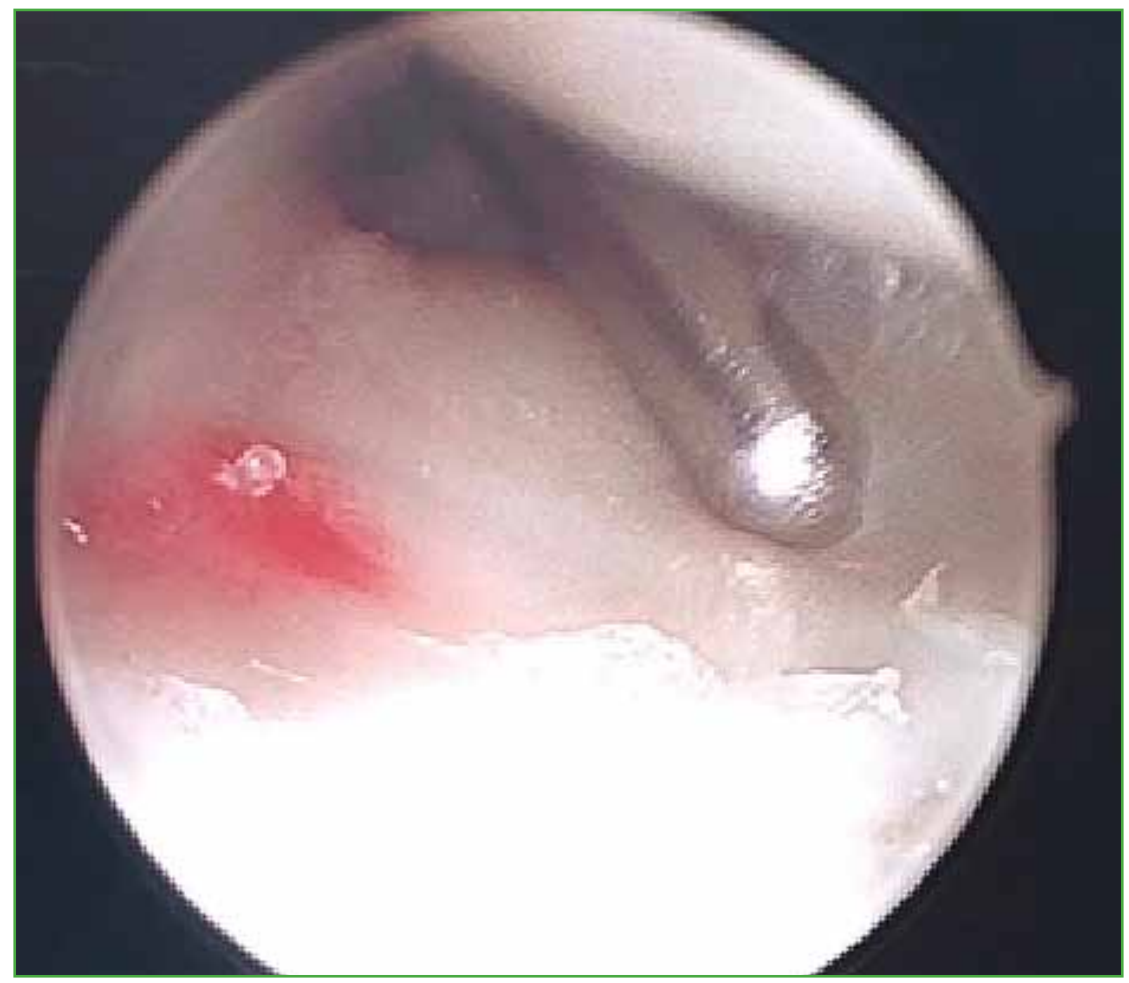

Figura 2. Desbridamiento del complejo del fibrocartílago triangular.

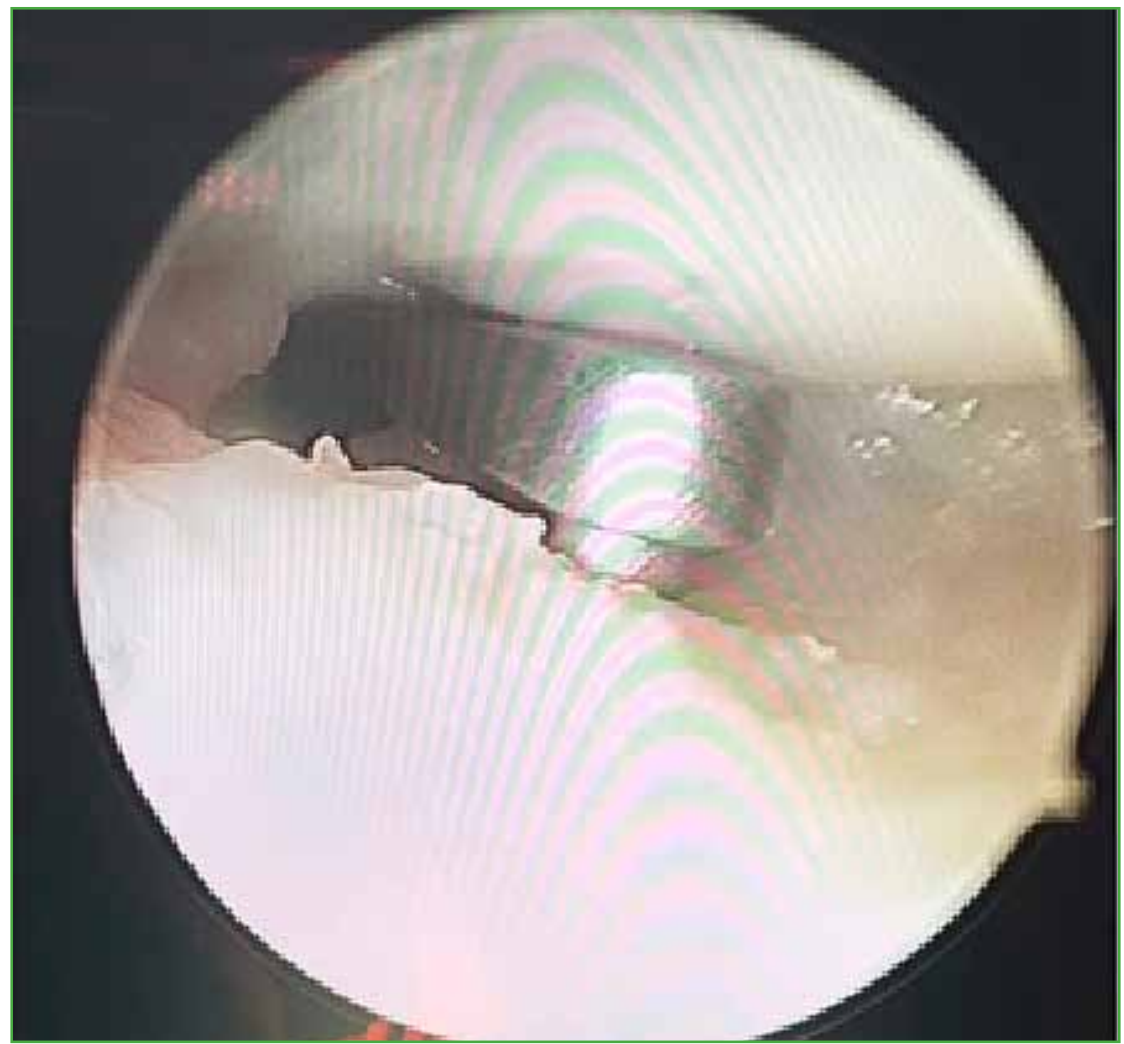

Figura 3. Guía del complejo del fibrocartílago triangular en posición 2/2. 


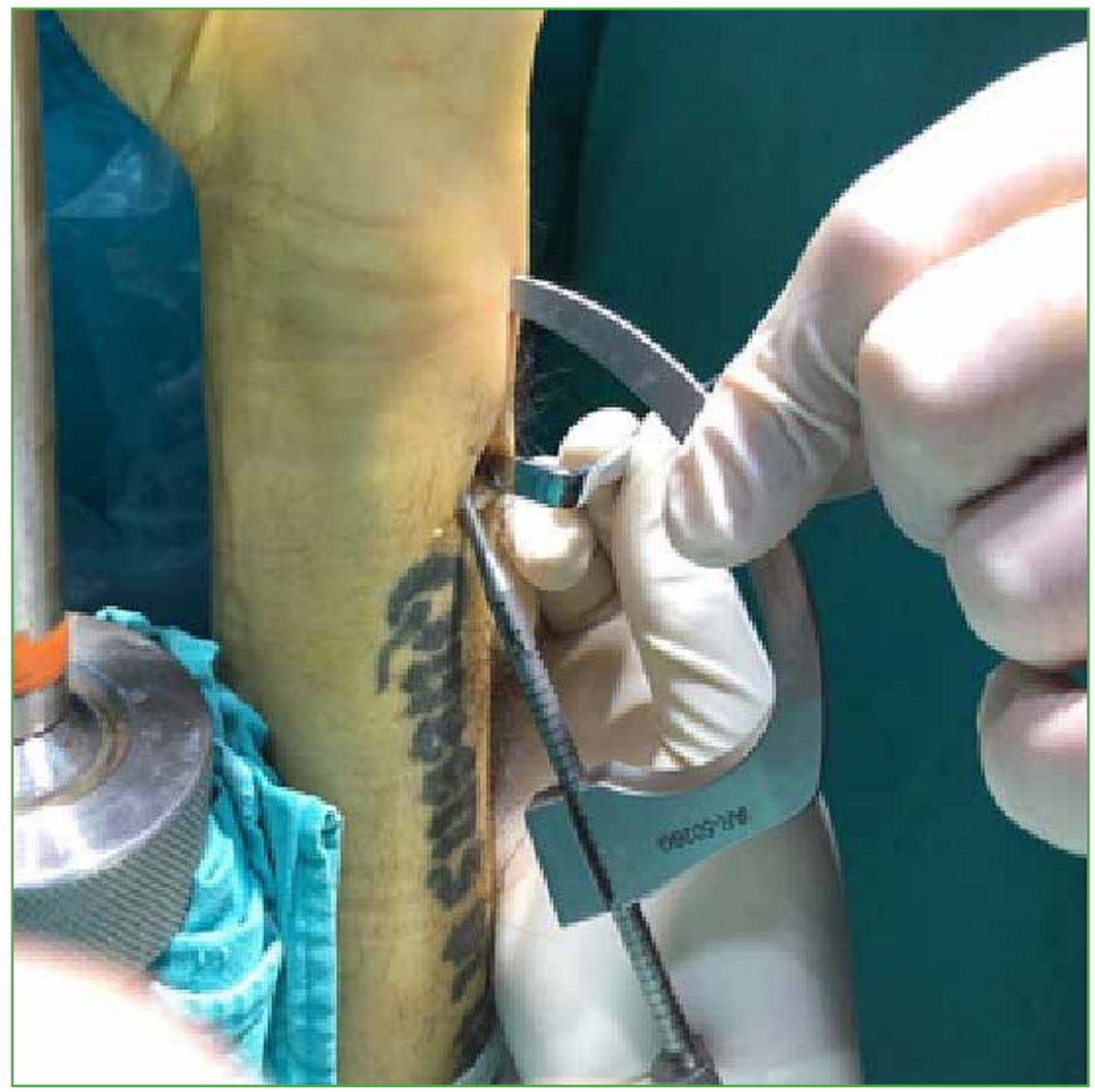

Figura 4. Colocación de la guía por el portal 6R e incisión cubital.

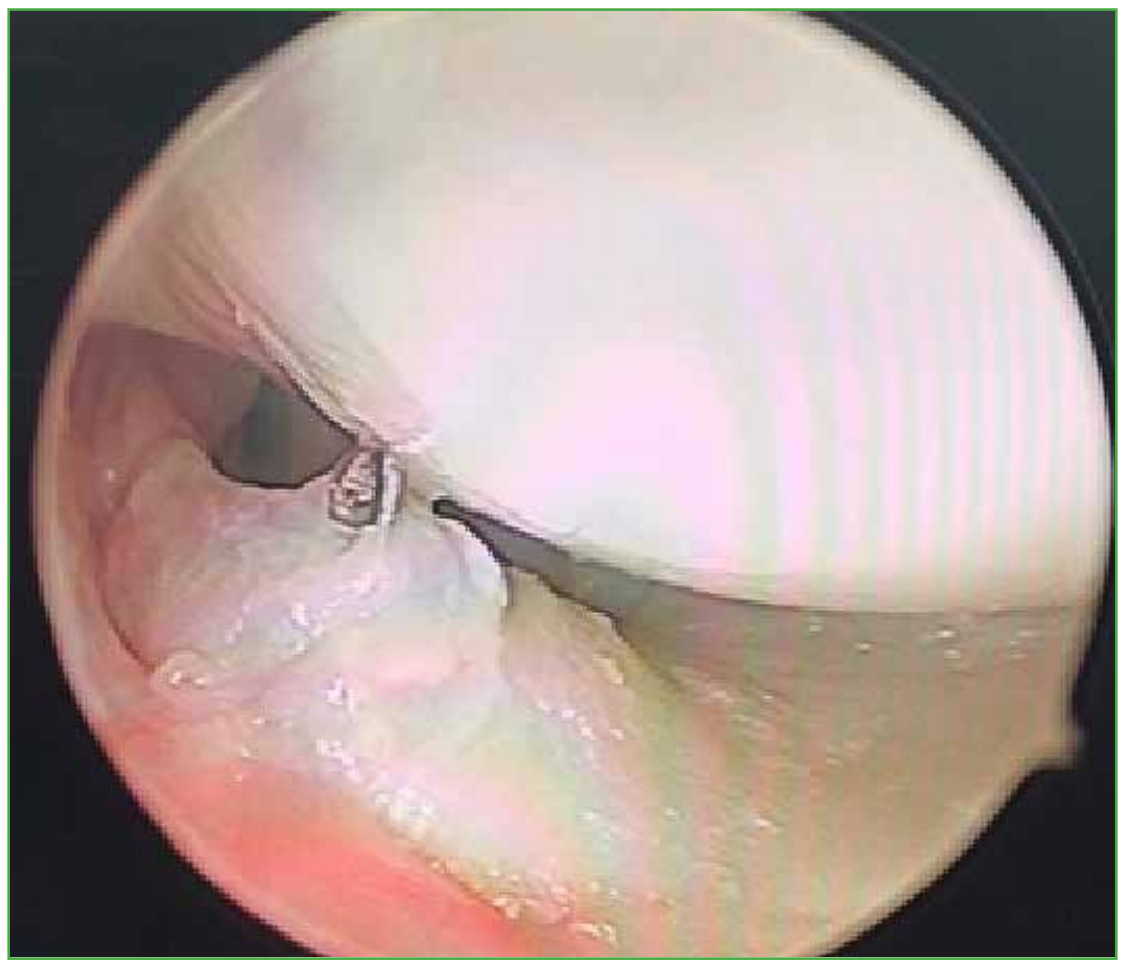

Figura 5. Clavija saliendo por el complejo del fibrocartílago triangular desde el túnel cubital. 


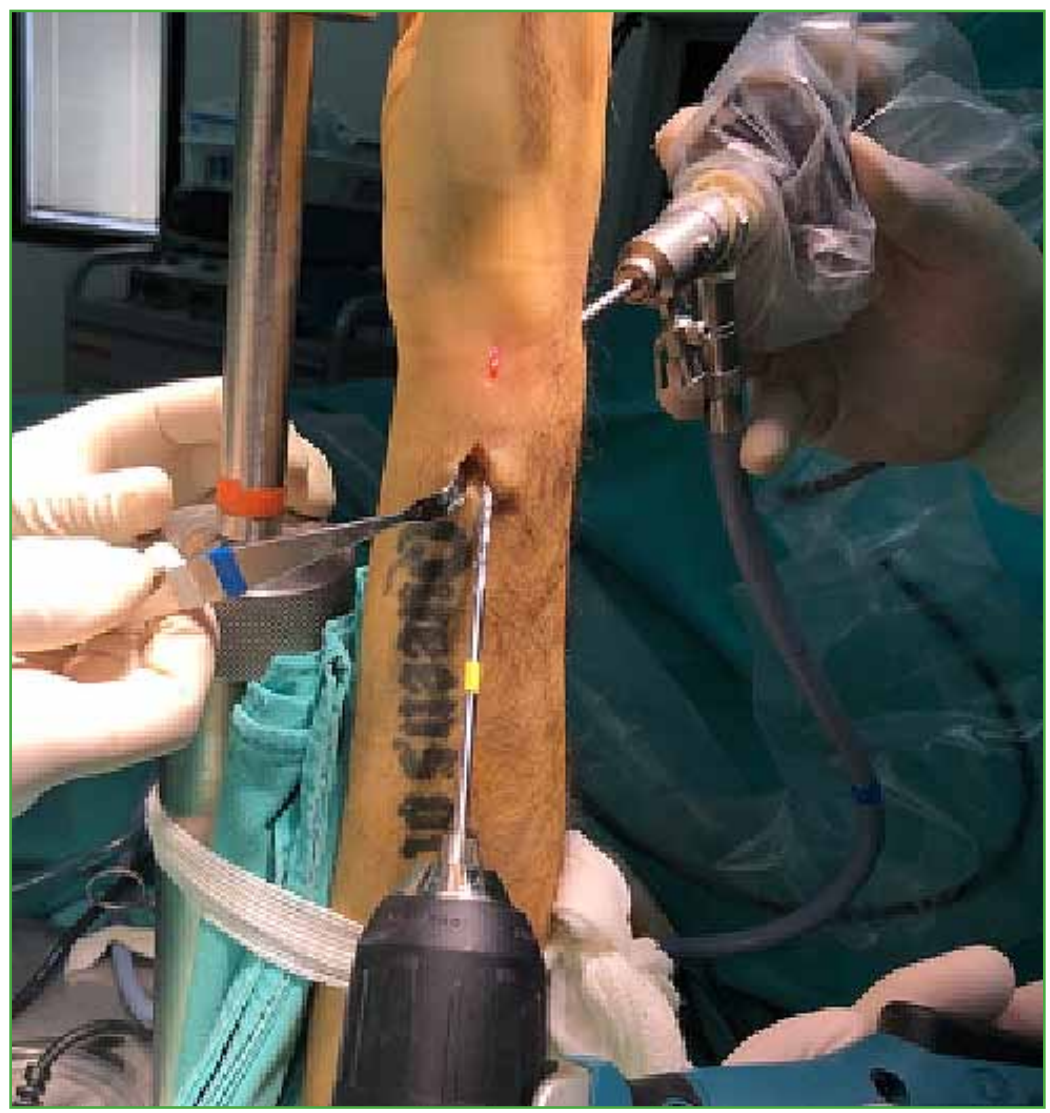

Figura 6. Colocación de la fresa por el cúbito para crear el túnel cubital.

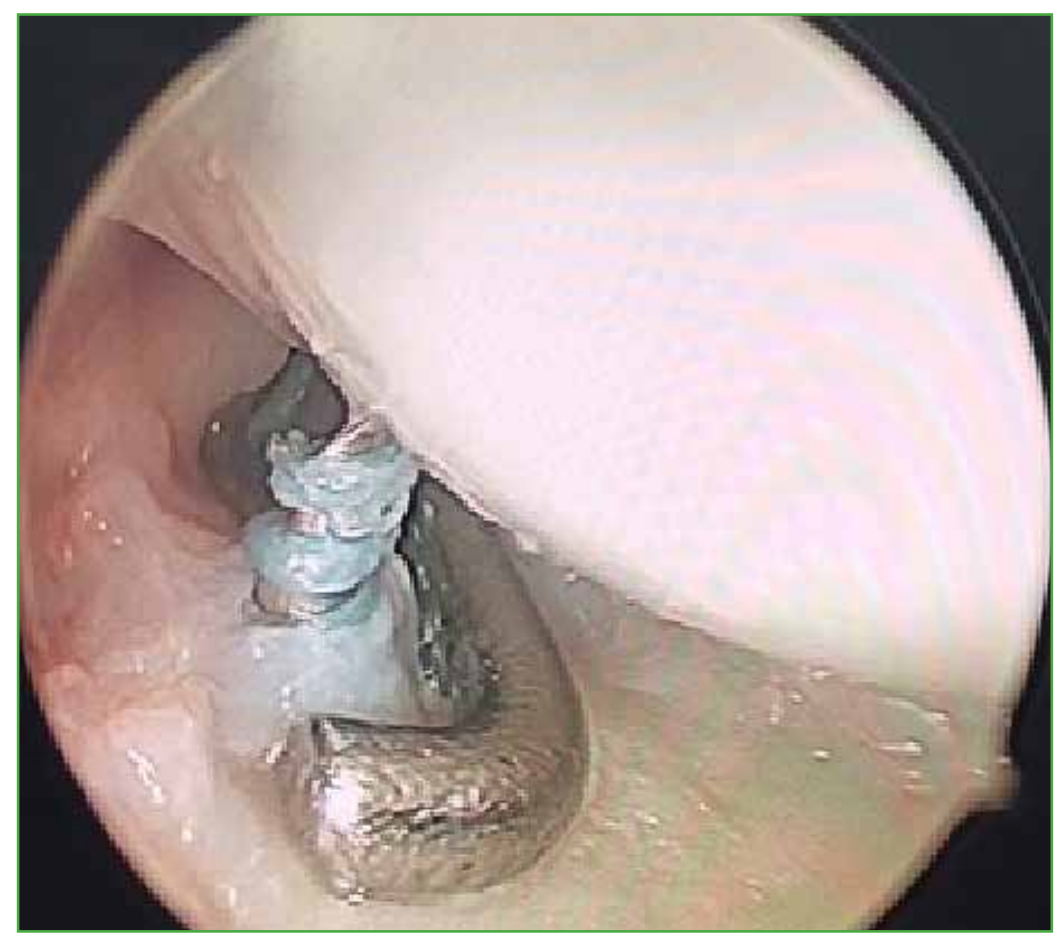

Figura 7. Pasaje del pasador de hilo con la sutura penetrando el complejo del fibrocartílago triangular. 


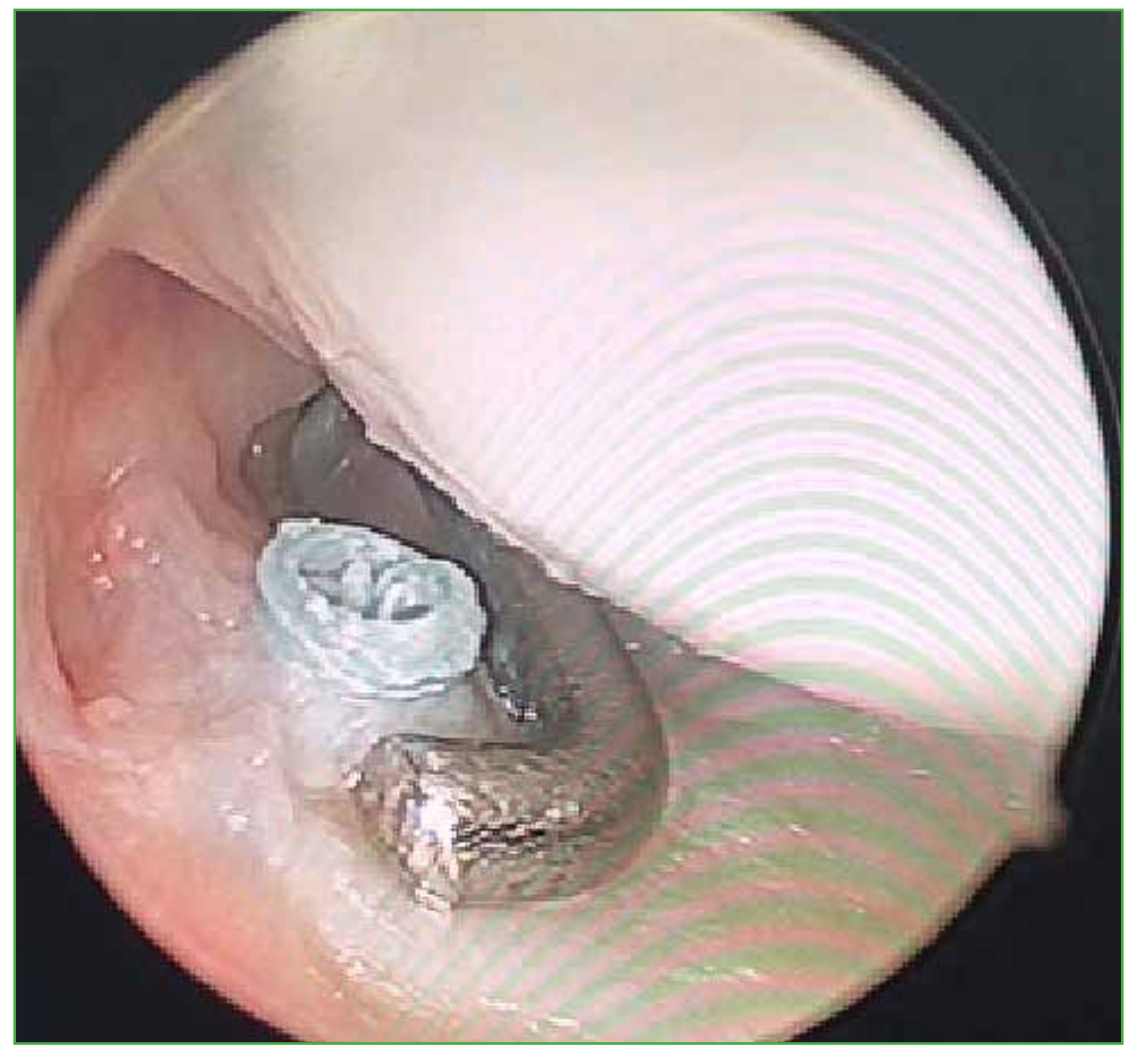

Figura 8. Bucle del hilo pasando por el complejo del fibrocartílago triangular.

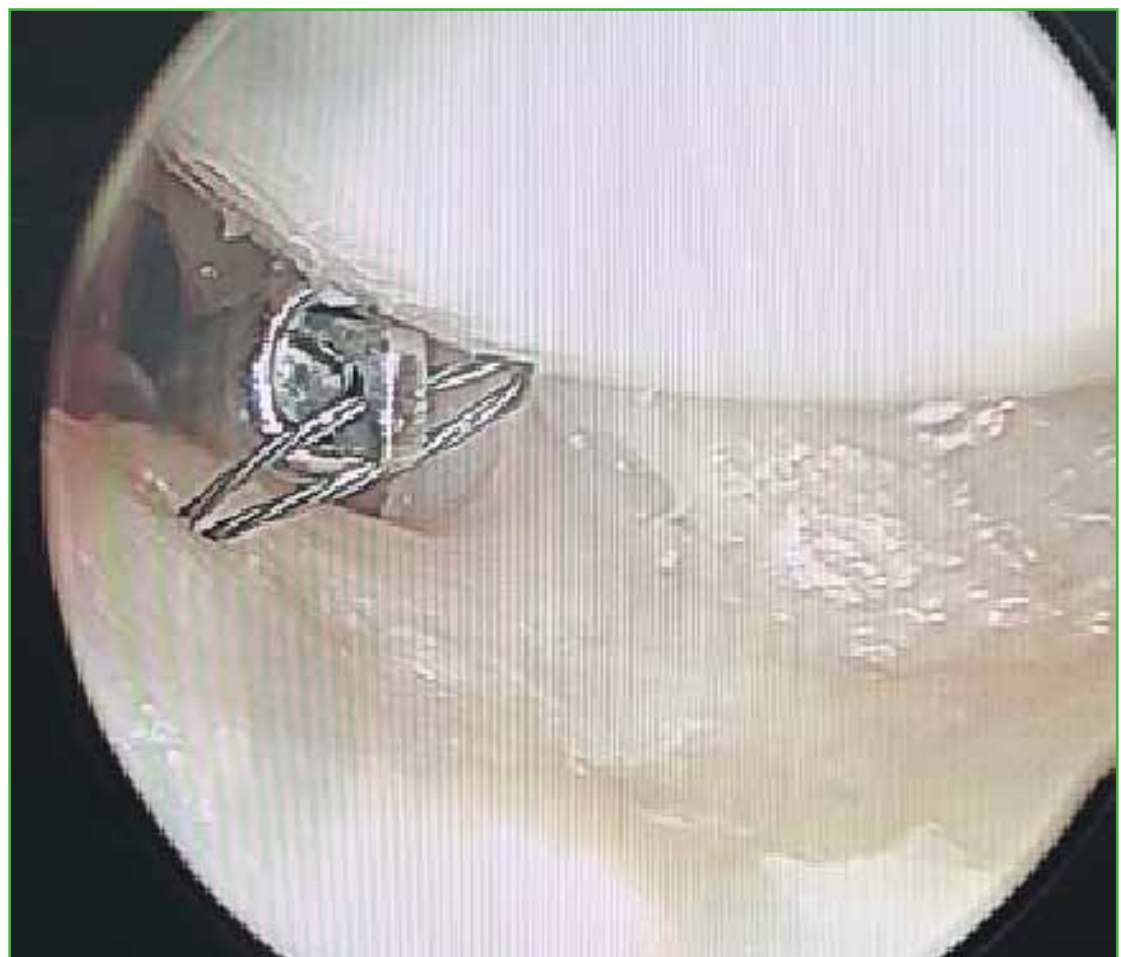

Figura 9. Pasador de sutura penetrando el complejo del fibrocartílago triangular con el rescatador de hilo. 


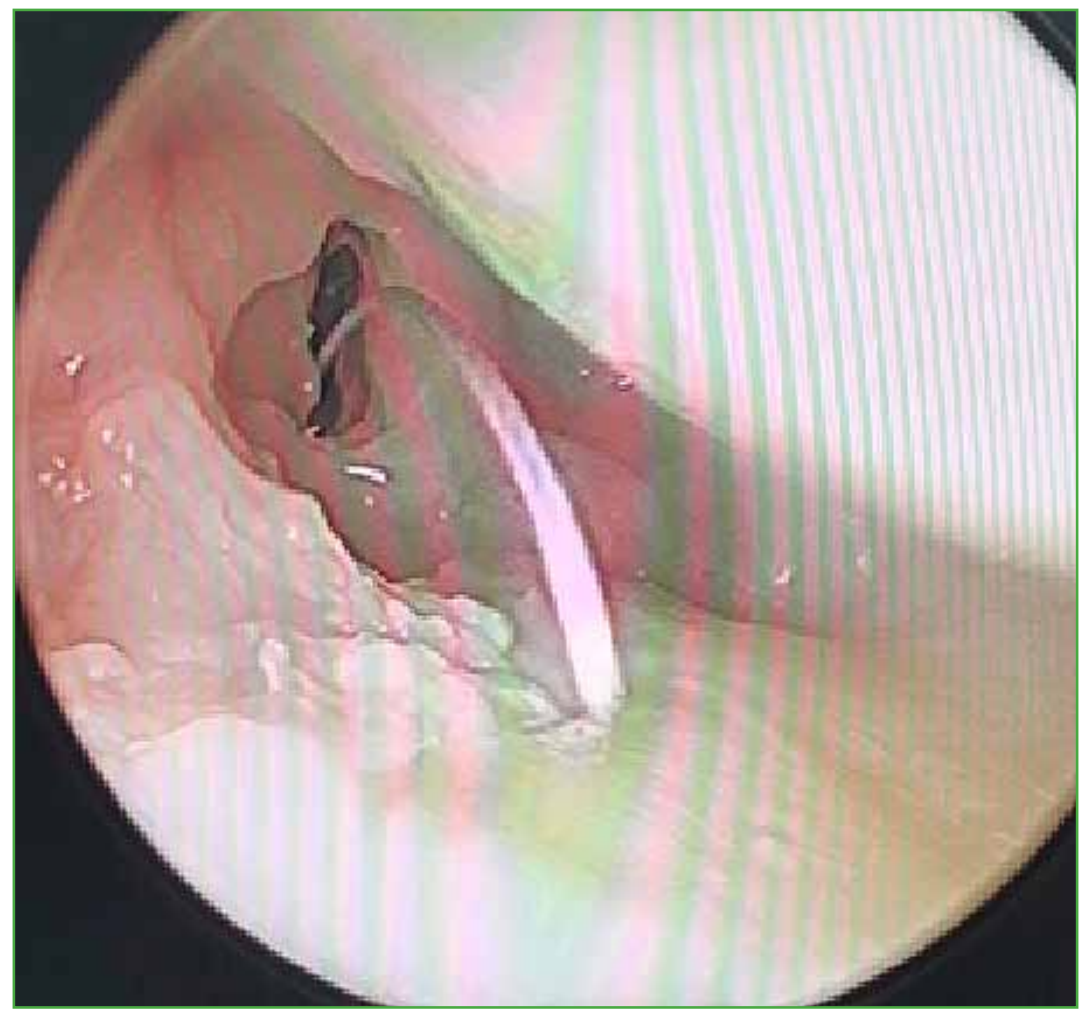

Figura 10. Final del pasaje del hilo pasando desde el portal 6R hacia el túnel cubital.

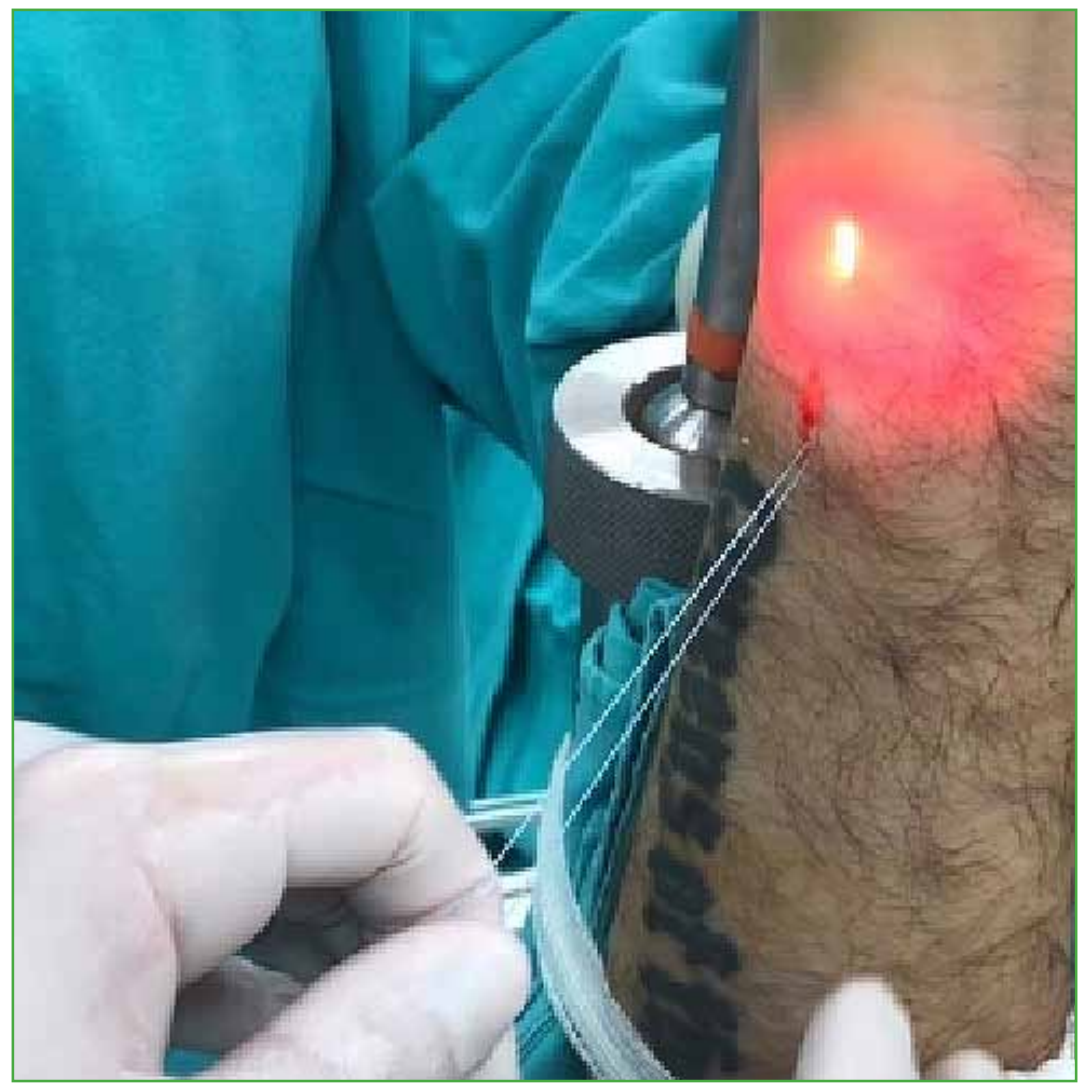

Figura 11. Los dos hilos rescatados por el túnel cubital. 


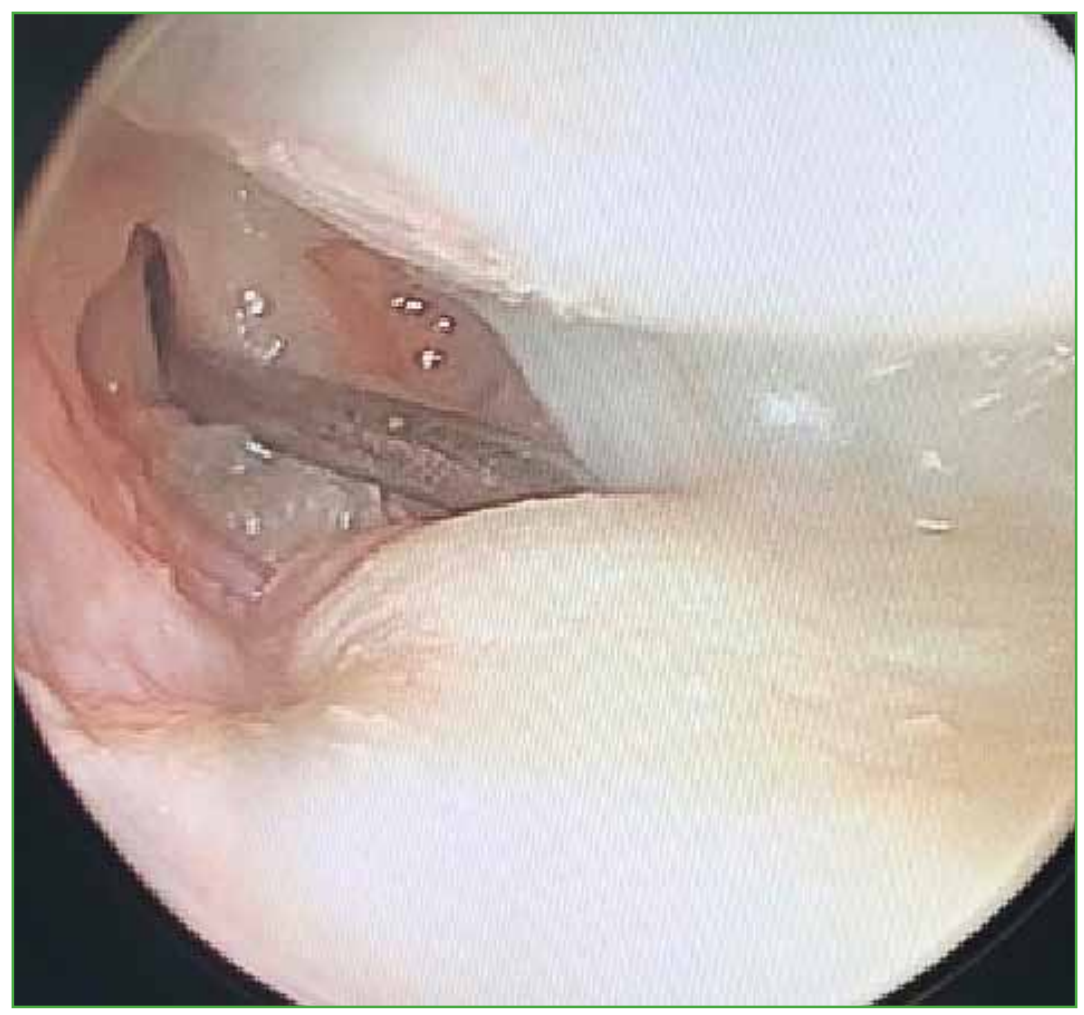

Figura 12. Negativización de las pruebas foveales al tensar los hilos.

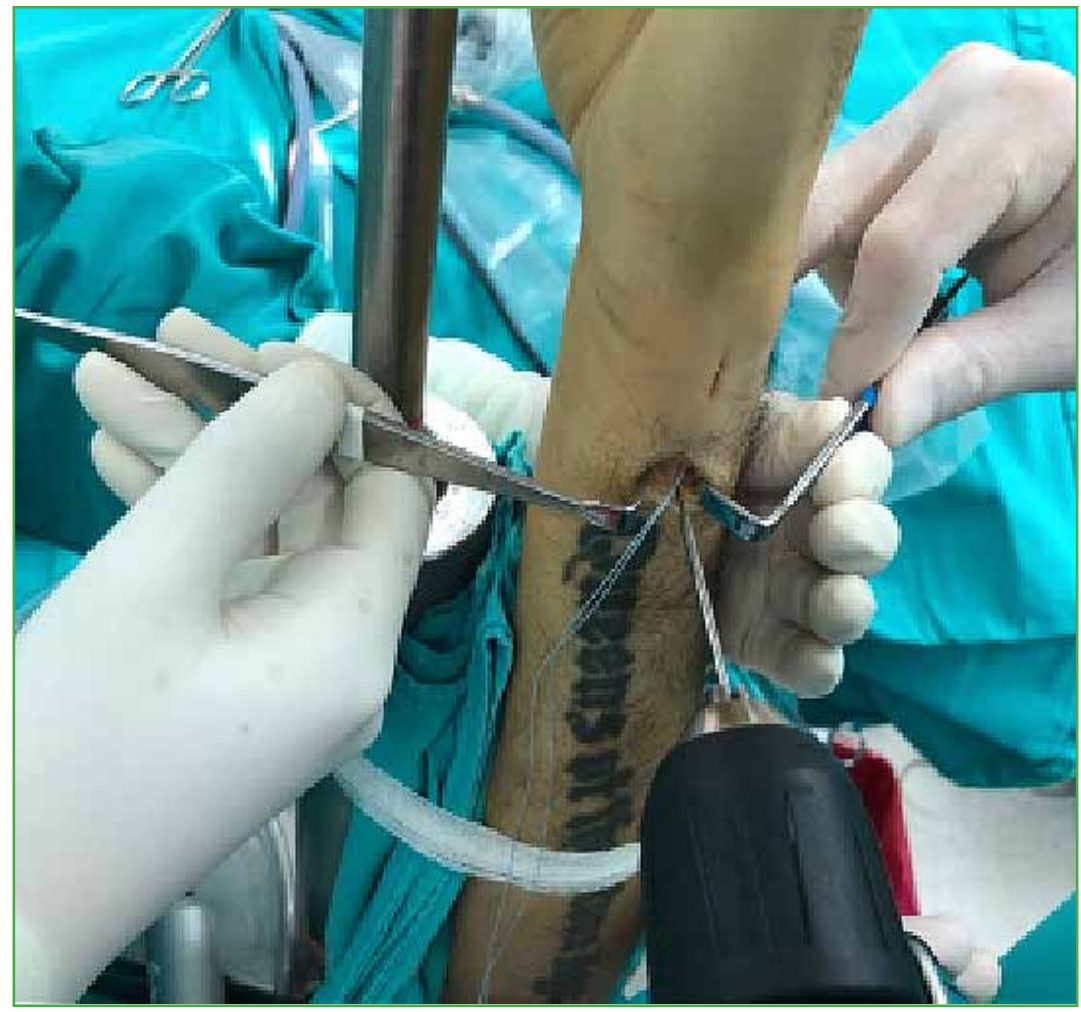

Figura 13. Fresado distal al túnel cubital para la colocación del anclaje sin nudo. 


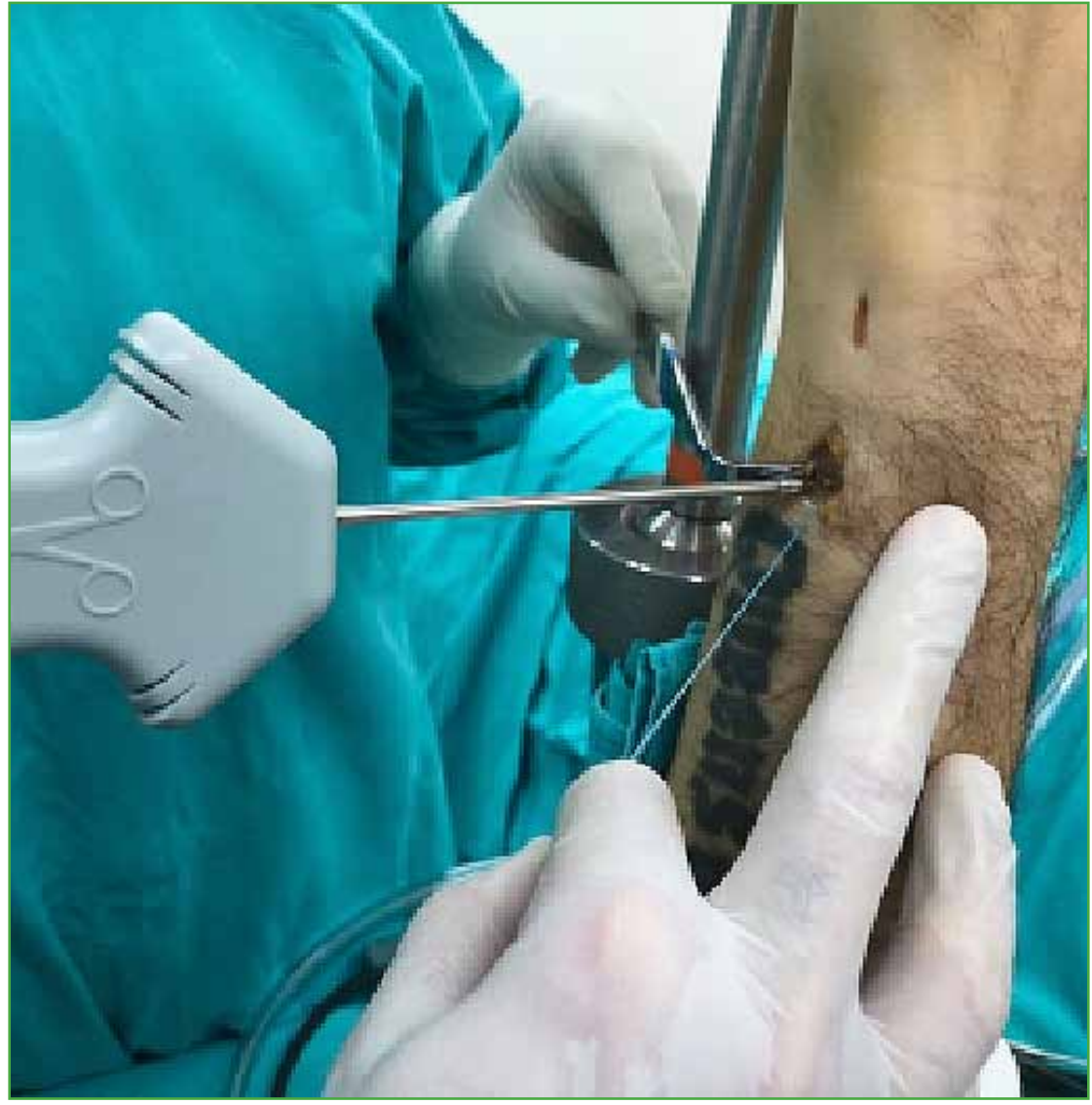

Figura 14. Colocación del anclaje sin nudo manteniendo la tensión de los hilos.

\section{RESULTADOS}

El período de seguimiento de los pacientes evaluados en forma retrospectiva osciló entre 12 y 26 meses, con un promedio de 18 meses. El tiempo entre la lesión y la cirugía fue inferior a tres meses (etapa aguda) en el 58,3\% y más de tres meses (etapa crónica) en el 41,7\%. Los arcos de movilidad de la muñeca se midieron con un goniómetro de mano PVS estándar. Los valores obtenidos fueron: $85^{\circ}$ de flexión, $70^{\circ}$ de extensión, $20^{\circ}$ de desviación radial y $30^{\circ}$ de desviación cubital. La fuerza de puño fue evaluada con un dinamómetro hidráulico estándar y se obtuvo un $70 \%$ de fuerza en comparación con la mano contralateral. El puntaje preoperatorio promedio del cuestionario DASH fue 56 (rango 41-90) y 8 (rango 2-16) en el posoperatorio. Los valores de la escala de muñeca de la Clínica Mayo fueron excelentes en el $83 \%$ y buenos en el 17\%, no hubo resultados malos. La escala analógica visual arrojó 8 puntos (rango 6-10) antes de la cirugía y de 2 puntos (rango 1-3) al final del seguimiento. Todos recuperaron la estabilidad articular evaluada mediante la prueba de Nakamura comparada con la muñeca sana.

Todos los pacientes deportistas operados reanudaron su actividad deportiva sin dificultades, al sexto mes de la cirugía.

En cuanto a las lesiones asociadas artroscópicas, hubo un 30\% de lesiones escafolunares (6 Geissler 1, 2 Geissler 2 y 2 Geissler 4) y un $20 \%$ de lesiones lunopiramidales ( 3 Geissler 2 y 2 Geissler 1). Todas las lesiones asociadas fueron reparadas en el mismo acto quirúrgico.

No hubo infecciones en la población estudiada. El 16\% de los pacientes tuvo parestesias en el territorio dorsal sensitivo del nervio cubital, que desaparecieron de manera espontánea. No fue necesario volver a operar a ningún paciente. 


\section{DISCUSIÓN}

Se desarrolló un estudio retrospectivo para analizar los resultados quirúrgicos en 12 pacientes adultos consecutivos con lesiones profundas del CFCT Atzei 2/3, tratados mediante anclaje artroscópico sin nudo. El $83 \%$ obtuvo resultados excelentes y el $17 \%$, buenos, según el puntaje de la escala de muñeca de la Clínica Mayo y el puntaje DASH promedio final fue 8. Todos se reincorporaron al deporte sin molestias al sexto mes de la cirugía.

Se han publicado diversas alternativas para solucionar este problemático cuadro.

Nakamura y cols. introducen la técnica afuera adentro mediante dos túneles separados para las lesiones foveales. En su estudio de 24 muñecas y un seguimiento de 3.5 años, obtuvieron resultados excelentes en el 54\%, buenos en el $12 \%$, satisfactorios en el $16 \%$ y malos en el 16\%, según la escala de muñeca de la Clínica Mayo. ${ }^{1}$ Shinohara y cols. presentan su estudio sobre reparación foveal mediante dos túneles y agujas en 11 pacientes con un seguimiento de 30 meses. Obtuvieron una fuerza de puño del $84 \%$ y resultados excelentes en el 63\%, buenos en el 27\% y malos en el 10\%, según la escala de muñeca de la Clínica Mayo. El 27\% tuvo neuropraxia en el territorio del nervio cubital rama sensitiva.

En el estudio de Ma y cols., se compararon los resultados biomecánicos entre la reparación abierta y la artroscópica en modelos cadavéricos, y se lograron mejores resultados de fuerza y una traslación cubital menor con las reparaciones artroscópicas. ${ }^{3}$ Atzei y cols. realizaron una artroscopia en 48 pacientes mediante un arpón foveal, y los resultados en la escala de muñeca de la Clínica Mayo fueron entre excelentes y buenos en el $83 \%$ de los casos; el $85 \%$ de los pacientes reanudó la práctica deportiva en el nivel previo a la lesión, aunque un $8 \%$ siguió teniendo una prueba de Nakamura positiva. El rango de movilidad obtenido fue del $95 \%$ para la flexo-extensión y del $98 \%$ para la pronosupinación, mientras que la fuerza de puño fue del $92 \%$ con un $10 \%$ de neuropatías sensitivas cubitales. ${ }^{1}$

Jung y cols. publicaron su estudio de reparación foveal artroscópica mediante anclaje sin nudo en 42 pacientes con un seguimiento de 26 meses. Los resultados en la escala de muñeca de la Clínica Mayo fueron excelentes (30\%), buenos (40\%), aceptables (26\%) y malos (4\%), la fuerza de puño obtenida fue del $69 \% .{ }^{8}$ Park comunica su estudio sobre reparación artroscópica foveal del CFCT mediante un túnel en 17 pacientes con un seguimiento de 30 meses. La fuerza de puño alcanzada fue del 57\% y los resultados fueron excelentes y buenos en el $83 \%$ de los pacientes evaluados con la escala de muñeca de la Clínica Mayo, el puntaje del cuestionario DASH fue 35,5 antes de la cirugía y 9 después de la intervención. ${ }^{9}$ Los datos comparativos de nuestra serie y las principales series publicadas se resumen en la Tabla 4.

Tabla 4. Resultados comparativos con las series de referencia

\begin{tabular}{|c|c|c|c|c|c|c|c|c|}
\hline Resultados & Técnica & $\begin{array}{c}\text { Pacientes } \\
\text { (n) }\end{array}$ & $\begin{array}{l}\text { Segui- } \\
\text { miento } \\
\text { (meses) }\end{array}$ & $\begin{array}{c}\text { Puño } \\
(\%)\end{array}$ & $\begin{array}{c}\text { Puntaje } \\
\text { DASH }\end{array}$ & $\begin{array}{l}\text { Escala de muñeca de la } \\
\text { Clínica Mayo (buenos } \\
\text { o excelentes) }(\%)\end{array}$ & $\begin{array}{l}\text { Neuro- } \\
\text { patía } \\
\text { sensitiva }\end{array}$ & $\begin{array}{l}\text { Inestabilidad } \\
\text { residual } \\
\text { (n) }\end{array}$ \\
\hline $\begin{array}{l}\text { Jung } \\
\text { y cols. }\end{array}$ & $\begin{array}{l}\text { Anclaje } \\
\text { sin nudo, } \\
1 \text { túnel }\end{array}$ & 42 & 26 & 70 & - & 71 & - & 2 \\
\hline $\begin{array}{l}\text { Shinohara } \\
\text { y cols. }\end{array}$ & $\begin{array}{l}\text { Anclaje } \\
2 \text { túneles } \\
\text { con aguja }\end{array}$ & 11 & 30 & - & - & 93 & 27 & - \\
\hline $\begin{array}{l}\text { Atzei } \\
\text { y Luchetti }\end{array}$ & $\begin{array}{l}\text { Anclaje } \\
\text { con arpón }\end{array}$ & 48 & 30 & 92 & $\begin{array}{c}42 \\
\text { precirugía } \\
20 \\
\text { poscirugía }\end{array}$ & 83 & - & 2 \\
\hline $\begin{array}{l}\text { Nakamura } \\
\text { y cols. }\end{array}$ & $\begin{array}{l}2 \text { túneles } \\
\text { con aguja }\end{array}$ & 24 & 42 & - & - & 66 & - & - \\
\hline $\begin{array}{l}\text { Park } \\
\text { y cols. }\end{array}$ & $\begin{array}{l}\text { Anclaje } \\
\text { sin nudo } \\
1 \text { túnel }\end{array}$ & 17 & 30 & $57 \%$ & $\begin{array}{c}35 \\
\text { precirugía } \\
7 \\
\text { poscirugía }\end{array}$ & 83 & - & - \\
\hline $\begin{array}{l}\text { Sala } \\
\text { y cols. }\end{array}$ & $\begin{array}{l}\text { Anclaje } \\
\text { óseo sin } \\
\text { nudo, } \\
1 \text { túnel }\end{array}$ & 12 & 15 & 70 & $\begin{array}{c}56 \\
\text { precirugía } \\
8 \\
\text { poscirugía }\end{array}$ & 100 & 16 & 0 \\
\hline
\end{tabular}


Todos estos autores han obtenido resultados buenos o excelentes similares a los nuestros, pese a que el seguimiento de nuestro estudio es más corto. A su vez, obtuvimos una fuerza de puño superior a la de la mayoría de los autores y un retorno deportivo de todos nuestros pacientes. Con los avances de la tecnología y los estudios biomecánicos del CFCT, sabemos que su estabilidad y la ARCD dependen de sus fibras foveales. Las reparaciones artroscópicas se pueden realizar mediante arpones, suturas con dos túneles, un túnel o sin túnel. Lo importante es dejar una ARCD estable sin dolor y funcional. ${ }^{1-3,14}$

Sobre la base de los estudios mencionados y de nuestra serie de casos, consideramos que la técnica artroscópica para la reparación foveal es fiable y que el anclaje óseo sin nudo sería la mejor opción biomecánica para su reparación.

Creemos que el anclaje mediante túneles es superior, desde el punto de vista biomecánico, al anclaje con un arpón foveal, ya que el uso de la guía permite realizar el túnel en zona 2/2 o 2/4 de Matsumoto, haciendo posible un anclaje ligamentario con traslación mínima de la sutura hasta su cicatrización y que evita una cicatrización ligamentaria elongada.

La colocación de un arpón en zona 2/2 o 2/4 requiere el uso de portales accesorios y nudos accesorios que complican técnicamente la cirugía y prolongan el tiempo quirúrgico. ${ }^{15}$

Las fortalezas de nuestro estudio son la valoración de pacientes con un cuadro homogéneo, el seguimiento mínimo acorde y el tratamiento realizado por un mismo cirujano, con un mismo método. Consideramos que las debilidades fueron el carácter retrospectivo de la serie y la muestra acotada de casos, y también la falta de un seguimiento a largo plazo. No tuvimos un grupo de control para demostrar la ventaja por sobre otras técnicas y creemos que las lesiones asociadas agregan heterogeneidad a la muestra y pueden afectar los resultados.

\section{CONCLUSIÓN}

La reparación artroscópica de las lesiones Atzei 2/3 mediante anclaje óseo sin nudo logró resultados funcionales aceptables y reproducibles técnicamente, mejorando los rangos de movilidad, recuperando la estabilidad de la ARCD en todos los casos y la fuerza de puño.

Conflicto de intereses: Los autores no declaran conflictos de intereses.

\section{BIBLIOGRAFÍA}

1. Atzei A, Luchetti R, Braidotti F. Arthroscopic foveal repair of the triangular fibrocartilage complex. J Wrist Surg 2015;4:22-30. https://doi.org/10.1055/s-0035-1544226

2. Trehan SK, Wall LB, Calfee RP, Shen TS, Dy CJ, Yannascoli SM, et al. Arthroscopic diagnosis of the triangular fibrocartilage complex foveal tear: a cadaver assessment. J Hand Surg Am 2018;43(7):680.e1-680.e5. https://doi.org/10.1016/j.jhsa.2017.12.017

3. Ma CH, Lin TS, Wu CH, Li DY, Yang SC, Tu YK. Biomechanical comparison of open and arthroscopic transosseous repair of triangular fibrocartilage complex foveal tears: a cadaveric study. Arthroscopy 2017;33(2):297-304. https://doi.org/10.1016/j.arthro.2016.10.027

4. Hong Li, Yujie Zhao, Yinghui Hua, Qianru Li, Hongyun Li, Shiyi Chen. Knotless anchor repair produced similar favourable outcomes as knot anchor repair for anterior talofibular ligament repair. Knee Surg Sports Traumatol Arthosc 2020;28(12):3987-93. https://doi.org/10.1007/s00167-020-05998-3

5. Lacheta L, Dekker TJ, Anderson N, Goldenberg B, Millett PJ. Arthroscopic knotless, tensionable all-suture anchor Bankart repair. Arthrosc Technic 2019;8(6):e647-e653. https://doi.org/10.1016/j.eats.2019.02.010

6. Palmer AK. Triangular fibrocartilage complex lesions: a classification. Hand Surg Am1989;14(4):594-606. https://doi.org/10.1016/0363-5023(89)90174-3 
7. Shinohara T, Tatebe M, Okui N, Yamamoto M, Kurimoto S, Hirata H. Arthroscopically assisted repair of triangular fibrocartilage complex foveal tears. J Hand Surg Am 2013;38(2):271-7. https://doi.org/10.1016/j.hsa.2012.11.008

8. Jung HS, Song KS, Jung HS, Yoon BI, Lee JS, Park MJ. Clinical outcomes and factors influencing these outcome measures resulting in success after arthroscopic transosseous triangular fibrocartilage complex foveal repair. Arthroscopy 2019;35(8):2322-30. https://doi.org/10.1016/j.arthro.2019.03.060

9. Park JH, Kim D, Park JW. Arthroscopic one-tunnel transosseous foveal repair for triangular fibrocartilage complex (TFCC) peripheral tear. Arch Orthop Trauma Surg 2018;138(1):131-8. https://doi.org/10.1007/s00402-017-2835-3

10. Geissler WB. Management of scapholunate instability. En: Geissler WB (ed). Wrist arthroscopy. New York: Springer; 2005:86-93.

11. De Smet L. The DASH questionnaire and score in the evaluation of hand and wrist disorders. Acta Orthop Belg 2008;74(5):575-81. PMID: 19058688

12. Cáceres-Palou E, López-Prats F, Mesa-Ramos M, et al. Valoración de resultados en Cirugía Ortopédica y Traumatología. Rev Esp Cir Ortop Traumatol 2005;49(S1):119-42. Disponible en: https://www.elsevier.es/enrevista-revista-espanola-cirugia-ortopedica-traumatologia-129-articulo-valoracion-resultados-cirugia-ortopedicatraumatologia-X1888441505011090

13. DaSilva MF, Goodman AD, Gil JA, Akelman EJ. Evaluation of ulnar-sided wrist pain. Am Acad Orthop Surg 2017;25(8):e150-e156. https://doi.org/10.5435/JAAOS-D-16-00407

14. Matsumoto T, Tang P, Fujio K, Strauch RJ, Rosenwasser MP. The optimal suture placement and bone tunnels for TFCC repair: a cadaveric study. J Wrist Surg 2018;7(5):375-81. https://doi.org/10.1055/s-0038-1661361

15. Chen WJ. Arthroscopically assisted transosseous foveal repair of triangular fibrocartilage complex. Arthrosc Tech 2017;6(1):e57-e64. https://doi.org/10.1016/j.eats.2016.09.004 